\title{
Aufklärung über die Aufklärung?
} Anmerkungen zu Jung-Stillings Geisterkunde (1808)

Les Lumières allemandes éclairées par les spectres? A propos de Geisterkunde de Jung-Stilling (1808)

Enlightenment about spirits? Remarks on Jung- Stillings Geisterkunde (1808)

\section{Wolfgang Fink}

\section{CpenEdition}

\section{Journals}

Édition électronique

URL : https://journals.openedition.org/ceg/10531

DOI : 10.4000/ceg.10531

ISSN : 2605-8359

Éditeur

Presses Universitaires de Provence

Édition imprimée

Date de publication : 1 octobre 2012

Pagination : 265-284

ISBN : 0751-4239

ISSN : 0751-4239

Référence électronique

Wolfgang Fink, „Aufklärung über die Aufklärung?

Anmerkungen zu Jung-Stillings Geisterkunde (1808)“, Cahiers d'Études Germaniques [Online], 63 | 2012,

Online erschienen am: 07 September 2020, abgerufen am 07 Juni 2021. URL: http://

journals.openedition.org/ceg/10531; DOI: https://doi.org/10.4000/ceg.10531 


\title{
Aufklärung über die Aufklärung? Anmerkungen zu Jung-Stillings Geisterkunde (1808) \\ Wolfgang FINK \\ Université Lumière Lyon 2
}

\begin{abstract}
Ehe man sich um das Wesen der Geister bekümmert, hat man erstlich die Existenz derselben, ob solche Substanzen, die keine Körper sind, vorhanden? zu untersuchen. Wer nicht offenbar leugnen will, dass ein Gott sei, noch den Menschen zu einer bloßen Maschine zu machen sich ansteht, der wird an der Existenz der Geister nicht den geringsten Zweifel haben $[\ldots]^{1}$.
\end{abstract}

Das institutionell abgesicherte normative Wissen $^{2}$ der deutschen Aufklärung, so wie es hier von Justus Christian Hennings in der Nachfolge von Johann Georg Walch vertreten wird, verbindet Zweifel an der Unsterblichkeit der menschlichen Seele systematisch mit dem Verdacht des Materialismus und damit des Atheismus ${ }^{3}$. Auch aufgeklärte Theologen wie der Neologe Wilhelm Abraham Teller rütteln nicht am Weiterleben nach dem Tode; Teller notiert in seinem in mancher Hinsicht so skandalträchtigen Wörterbuch des Neuen Testaments lakonisch und apodiktisch zugleich, der Begriff der Unsterblichkeit der Seele erfasse die "Bedeutung dessen, was von uns nach dem Tode weiterlebt", Damit wird das für die Aufklärung von Christian Wolff $^{5}$ bis $\mathrm{Kant}^{6}$ spezifische Triptychon von religiösem Glauben,

1 Artikel Geist in Johann Georg Walchs philosophisches Lexicon... fortgesetzt ... von Justus Christian Hennings, vierte Auflage Leipzig, 1775, Bd. I, S. 1516.

2 Michel FouCAUlT, L'archéologie du savoir, Paris 1969, S. 166.

3 Quirin HuONDER, Das Unsterblichkeitsproblem in der abendländischen Philosophie, Stuttgart, 1970

4 Wilhelm Abraham Teller, Notiz Seele, in Wörterbuch des Neuen Testaments zur Erklärung der christlichen Lehre, [1772], dritte Auflage bei August Mylius, 1780, S. 362.

5 Christian WOLFF, Vernünftige Gedanken von dem gesellschaftlichen Leben der Menschen und insbesondere dem gemeinen Wesen, ND München, 2004. Vgl. Christian LinK, Die Staatstheorie Christian Wolffs, in Werner SCHNEIDERS (Hg.), Christian Wolff, Hamburg, 1986, S. $180 \mathrm{ff}$. 
Moralität und Unsterblichkeit der Seele als Basis des individuellen und kollektiven Fortschritts bestätigt ${ }^{7}$. Theologie und Philosophie, Moral und Ethik trennen sich nicht; die Umwandlung des religiösen Pathos in Ethik ${ }^{8}$ wird immer wieder hinausgezögert - nicht zuletzt auf Grund der Rückbindung der Moral an die Unsterblichkeit der Seele.

Das Dogma der Unsterblichkeit behauptet sich auch in den Jahren 17751776, einem der zentralen Wendepunkte der theologischen Aufklärung: nach dem erfolgreich geführten bayrischen "Hexenkrieg" dominiert jetzt die Rückweisung der Existenz des Teufels die Debatten und vertieft die schon im Fahrwasser von Thomasius deutlich gewordenen Zweifel an dessen Einflussnahme auf das Handeln des Menschen. Direkt damit verbunden ist die Frage der Erbsünde, die ihre längst fällige Umdeutung erfährt ${ }^{10}$ : trotz der hinsichtlich der Moralität der Bevölkerung vorherrschenden Skepsis erarbeiten sich die Aufklärer die theologisch-philosophisch abgesicherte Gewissheit, dass der Mensch prinzipiell zu selbstständigem moralischem Handeln fähig ist. Gerade diese Umorientierung trägt nachdrücklich zur

6 Immanuel Kant, Die Religion in den Grenzen der bloßen Vernunft, [1793], Stuttgart, 1981; vgl. Ernst Cassirer, Kants Leben und Lehre, ND Darmstadt 1975.

7 Exemplarisch hierfür ist Moses Mendelssohn, der mit der Logik des ontologischen Gottesbeweises den Wunsch nach Unsterblichkeit als eine anthropologische Konstante versteht, also an Gott rückbindet. Und weil dieser Wunsch von Gott dem Menschen eingegeben wird, kann und muss er auch erfüllt werden: "O nein meine Freunde, nicht umsonst hat uns die Vorsehung ein Verlangen nach ewiger Glückseligkeit eingegeben; es kann und wird befriedigt werden. Das Ziel der Schöpfung dauert so lange, als die Schöpfung, die Bewunderer göttlicher Vollkommenheiten, so lange, als das Werk, in welchem diese Vollkommenheiten sichtbar sind. So, wie wir hiernieden dem Regenten der Welt dienen, indem wir unsere Fähigkeiten entwickeln: so werden wir auch in jenem Leben unter seiner göttlichen Obhut fortfahren, uns in Tugend und Weisheit zu üben, uns unaufhörlich vollkommener und tüchtiger zu machen [...]". Die Unsterblichkeit der Seele ist also eine metaphysische Evidenz, die von Theologie und Philosophie weiterzutragen ist. Mendelssohn betont die Weiter- und Höherentwicklung der Seelen bis ins Unendliche, etabliert also eine Kontinuität zwischen Diesseits und Jenseits, die durch den physischen Tod nur scheinbar unterbrochen wird. Keine Antwort gibt Mendelssohn jedoch auf die Frage, wo diese Weiterentwicklung stattfindet: "Wenn Jemand mich dieses fragt, so antworte ich: Freund, du forderst mehr, als meines Berufes ist. Ich habe dich durch alle Krümmungen des Labyrinths hindurch geführt, und zeige dir den Ausgang: hier endigt sich mein Beruf". Diese Vorsicht öffnet aber zugleich der Willkür Tür und Tor: "Wissen es unsere Dichter und Fabellehrer besser: so mögen sie andere davon versichern. Es schadet vielleicht nicht, wenn gewisser Leute Einbildungskraft auf eine solche Weise beschäftigt und angestrengt wird”. Moses MENDESSOHN, Phaedon oder über die Unsterblichkeit der Seele, [1767], Leipzig, o.J. [tredition - Verlag], Zitate S. 122 und S. 132.

8 Ernst CASSIRER, Die Philosophie der Aufklärung [1932], Hamburg, Felix Meiner, 1998, S. 219.

9 Vgl. Wolfgang BeHRINGer, "Der bayrische Hexenkrieg. Die Debatte am Ende der Hexenprozesse", in Sönke LORENZ / Dieter R. BAUER (Hg.), Das Ende der Hexenverfolgung, Stuttgart, Franz Steiner Verlag, 1995, S. 287-313. Zum letzten Hexenprozess im deutschen Kulturraum vgl. Jean MONDOT, "Sorcellerie, justice et opinion publique à la fin du XVIII siècle", in Thomas Nicklas (Hg.), Glaubensformen zwischen Volk und Eliten, Halle, Universitätsverlag, 2012, S. 101-113.

10 CASSIRER, Die Philosophie der Aufklärung, S.188. 
Entstehung der Volksaufklärung bei ${ }^{11}$, durch die nicht nur der Teufel, sondern auch die von den Geistern ${ }^{12}$ prinzipiell zu unterscheidenden Gespenster ${ }^{13}$ aus der Vorstellungswelt der ungebildeten Bevölkerung vertrieben werden sollen - auch hier eine Beschleunigung und Vertiefung langfristiger Tendenzen, denn die Existenz der Gespenster war spätestens seit 1761 und den "Erscheinungen" im Carolinum zu Braunschweig ${ }^{14}$ Objekt öffentlicher Kontroversen.

Diese Großoffensive gegen den Aberglauben ist umso bemerkenswerter, als sich die Autoren der Gefahr bewusst waren, dass sie ihr Publikum durch ihre Thesen aus Jahrhunderte alten Traditionen lösten und daher Gefahr liefen, sie intellektuell zu überfordern. Ausmaß und Methode der Volksaufklärung standen daher zur Debatte ${ }^{15}$ und auch an Protesten gegen diese Offensive mangelte es nicht. Jung-Stillings Erweckungsromane und seine Publizistik in den neunziger Jahren können als gezielte Gegenpädagogik verstanden werden, bezeichnete er sich doch selbst als "Missionarius in der Aufklärung"16. Die seiner Pädagogik zugrundeliegenden theologischen Überzeugungen kommen allerdings schon in seiner frühen Polemik gegen Nicolai zum Ausdruck, die er anschließend durch seine Kantlektüre theoretisch abzusichern versucht. Jung-Stillings Gegenoffensive kulminiert sozialgeschichtlich gesehen in seinem Engagement für die

11 Vgl. den Überblick von Wolfgang RUPPERT, Volksaufklärung im späten 18. Jahrhundert, in Rolf GRIMMINGER (Hg.), Hansers Sozialgeschichte der deutschen Literatur, Bd. III, 1, München, dtv, 1980, S. 341f.

12 Vgl. die Verbindung von Seele und Geist im Zedler: "Man nennt sie eine geistliche Substanz, und dies ist ihr gemeines Wesen, welches sie mit den andern Geistern gemein hat; und weil sie eine Art solcher Geister ist, kommt ihr alles dasjenige zu, was einem solchen Geist zukommt. Durch einen solchen Geist versteht man überhaupt eine Substanz, die von Materie nichts an sich hat, und mit Verstand und Wissen begabt ist; mithin muss man auch von der menschlichen Seele sagen, sie sei ein immaterielles Wesen, das nichts von der Materie an sich hat", Artikel "Seele, Anima", in Johann Heinrich Zedlers Universal-Lexicon, Leipzig, 1742, Bd. 36, Spalte 1060.

13 Die Erscheinung von Gespenstern beruht für den Zedler nicht immer auf Sinnestäuschung und/oder Manipulation. So heisst es unter explizitem Bezug auf Johann Georg Walch : "Was mich anbelangt, ob ich gleich in selben Fällen sehr argwöhnisch bin, und glaube das meiste davon Fabeln und Blendwerk sei; doch weil ich in solchen Sachen sehr fleissig nachgeforscht habe, so habe ich unfehlbare Zeugnisse von der Wahrheit solcher Erscheinungen bekommen, teils aus dem Mund ohnstreitig ehrlicher und frommer Leute, teils auch aus der Erzählung vieler Personen, die es selbst gehört oder gesehen", Ibid., Artikel "Spectrum", Bd. 38, Spalte 1381.

14 Vgl. das Nachwort des Herausgebers zu Georg Wilhelm Wegner, Philosophische Abhandlung von Gespenstern, Hannover, Wehrhahn, 2006. Zu dem die Debatten dominierenden Baumgarten -Schüler Meier vgl. Yvonne WüBBEN, Gespenster und Gelehrte. Die ästhetische Lehrprosa Georg Friedrich Meiers (1718-1777), Tübingen, Niemeyer, 2007.

15 Vgl. Rudolf Zacharias BECKER, Versuch über Aufklärung des Landmanns, Dessau und Leipzig, 1785, S. 36; Johann Wilhelm RECHE, Neuer Versuch über die Grenzen der Aufklärung, Düsseldorf, Joh. Christ. Dänzer, 1789, S. 180.

16 In einem Brief an einen unbekannten Adressaten vom 16. Dezember 1798. Zitiert nach Gerhard SCHWINGE, Jung-Stilling als Erbauungsschriftsteller der Erweckung, Göttingen, Vandenhoeck \& Ruprecht, 1994, S. 17. 
Erweckungsbewegung, theoretisch gesehen 1808 in der Geisterkunde, in der er nicht nur die Geister, sondern auch die Gespenster, die Hexen und nicht zuletzt den Teufel wieder zum Leben erweckt. Dies bedeutet jedoch nichts anderes als eine radikale Umkehrung der diskursiven Konstellation: gehörten die Gespenster noch in der Mitte des 18. Jahrhunderts zum institutionell abgesicherten Wissen der Aufklärung, so wird deren Existenz nun von JungStilling, einem dezidierten Aufklärungsgegner verteidigt, wodurch er seinerseits die Kritik der kirchlichen Institutionen auf sich zieht. Exemplarisch deutlich wird dies am Beispiel seiner Spekulationen über den Hades und der damit verbundenen These der Apokatastasis Panton, der "Lehre von der Reinigung nach dem Tod, und [...] Wiederbringung aller Dinge" ${ }^{17}$, die im Zentrum seines Konflikts mit der Deutschen Christentumsgesellschaft stehen wird. Um diesen Umschlag zu verdeutlichen, werde ich zunächst Jung-Stillings Polemik gegen Nicolai ansprechen (I), dann die Thesen seiner Theorie der Geisterkunde referieren (II) und abschließend einiges Aspekte des Erbes der Aufklärung, so wie es sich um 1810 darstellt, ansprechen (III).

\section{Jung-Stilling gegen Nicolai}

"Sebaldus Einwendung ist die Sprache aller Freigeister, die nichts für sündlich halten, als was just der menschlichen Gesellschaft zuwider sei" ${ }^{\prime \prime}$. Die in Nicolais Roman ${ }^{19}$ zum Ausdruck kommende Verbindung von neologischer Bibelkritik, sozialem Utilitarismus und urbanem Spott an philisterhaften Pietisten ${ }^{20}$ wird von Jung-Stilling nicht nur als individuelle Abwendung von der christlichen Religion verstanden, sondern auch als Bedrohung für die Gesellschaft aufgefasst, denn die Verspottung des Priesterstandes gilt ihm als "Gotteslästerung" und als Unterminierung der Moral $^{21}$, insbesondere der des "Pöbels" 22 . Der Kontroverse zwischen JungStilling und Nicolai liegen diametral entgegengesetzte anthropologische

17 Johann Heinrich JUNG-STILLING, Apologie der Geisterkunde, veranlasst durch ein über dieselbe abgefasstes Gutachten des hochwürdigen geistlichen Ministriums [sic] zu Basel, [1809], Nürnberg, Verlag der Raw'schen Buchhandlung, 1833, S. 55.

18 Johann Heinrich Jung-Stilling, Die Schleuder eines Hirtenknaben gegen den hohnsprechenden Philister, den Verfasser des Sebaldus Nothanker, [1775], in Johann Heinrich JunG-STILling, Sämmtliche Schriften, Ergänzungsband, Stuttgart, Scheible's Buchhandlung, 1838 , S. 721.

19 Friedrich NiCOLAI, Leben und Meinungen des Herrn Magister Sebaldus Nothanker, [1776]. Kritische Studienausgabe, Stuttgart, Reclam, 2001.

20 Albrecht BEUTEL, “Aufklärung und Pietismus auf dem Weg nach Berlin : Die Figur des Frömmlings in Friedrich Nicolais Roman 'Sebaldus Nothanker'”, in Zeitschrift für Theologie und Kirche 99, 2002, S. 262-277.

21 Jung-StiLling, Schleuder eines Hirtenknaben, S. 737.

22 Ibid., S. 744. 
Überzeugungen zugrunde ${ }^{23}$ : begründet Nicolai seinen Utilitarismus und seinen relativen Optimismus mit dem durch Erziehung erreichbaren und daher verbesserbaren kognitiven Potential des Menschen ${ }^{24}$, so beruft sich Jung-Stilling auf das Dogma der Erbsünde:

Erbsünde ist nach dem reinen Begriff eines Christen die von dem ersten
Menschen allen seinen Nachkommen angeerbte Unfähigkeit, zu der Bestimmung
zu gelangen, zu welcher der Mensch geschaffen ist, und eben die vom ersten
Menschen allen seinen Nachkommen angeborene Fähigkeit, das Tun und Lassen
nach den Reizen der Sinnlichkeit einzurichten ${ }^{25}$.

Daraus resultieren für ihn mehrere Konsequenzen: zunächst die Tatsache, dass die gesellschaftliche Entwicklung generell als die Geschichte einer Regression zu verstehen ist:

Die menschliche Natur ist ganz verdorben, d.i. die Menschen, sich selbst überlassen, wandeln ihre Wege fort, dergestalten, dasss sie, anstatt immer mehr Gott ähnlicher, mithin vollkommen glückselig zu werden, immer zurück bleiben, immer mehr und mehr ihre Seele mit vergänglichen Scheingütern sättigen $[\ldots]^{26}$.

Soweit die allgemeine Tendenz, die allein durch individuelle Erweckungserlebnisse minimal relativiert werden kann. Diese implizieren für Jung-Stilling die Einsicht in das eigene Fehlverhalten und die ihr zugrundeliegenden Karenzen der Vernunft:

Vor und nach wird ihm die Sache klar in seinem Gemüth, und was der natürlichen Vernunft unbegreiflich und gar ungereimt vorkam, das beginnt sie nun einzusehen; er verwundert sich, dass er so töricht gewesen $[\ldots]^{27}$.

Die Trennung von Vernunft und Glaube ist programmatisch. Fühlen und nicht "historisch glauben" lautet die Maxime; in der die deutsche Aufklärung dominierenden rationalen Tradition eines Christian Wolff ist laut JungStilling eine authentische Glaubens- und Gotteserfahrung unmöglich ${ }^{28}$, was wiederum bedeutet, dass die Offenbarung als Erkenntnisquelle über der

23 Rainer VINKE, Jung-Stilling und die Aufklärung. Die polemischen Schriften Johann Heinrich Jung-Stillings gegen Friedrich Nicolai (1775/76), Stuttgart, 1987, S. 157.

$24 \mathrm{Zu}$ Nicolai immer noch grundlegend: Horst MöLlER, Aufklärung in Preußen. Der Verleger, Publizist und Geschichtsschreiber Friedrich Nicolai, Berlin, 1974.

25 JUNG-STILling, Schleuder eines Hirtenknaben, S. 744.

26 Ibid., S. 722.

27 Ibid., S. 746. Schon hier wird die später für Jung-Stillings Argumentation so wichtige Rückbeziehung auf angeblich repräsentative persönliche Beobachtungen deutlich: "Mir sind viele dergleichen Exempel bekannt, unter andern ein Schreiner, den ich in der Kur gehabt, welcher über dem Saufen und Spielen plötzlich dergestalt über seine Sünden gerührt worden, dass er wie tot zur Erde gefallen, und von der Zeit an ein anderer Mensch geworden”, Ibid., S. 726 .

28 VINKE, Jung-Stilling und die Aufklärung, S. 148. 
Vernunft steht. Deshalb notiert er, hier mit der lutheranerischen Orthodoxie konvergierend ${ }^{29}$ :

Ist die Bibel ganz Offenbarung, so haben wir keinen Streit; ist sie nur zum Teil Offenbarung, und das, was Offenbarung ist, soll mit der Vernunft herausgesucht werden, so ist die Vernunft über die Offenbarung, und wir haben keine nötig; ist sie gar nicht Offenbarung, so ist wieder nichts zu streiten ${ }^{30}$.

Kein dritter Weg zwischen Christen und Deisten, zwischen Christen und Neologen; laut Jung-Stilling muss der Gläubige "genau bei der Lehre Christi und seiner Apostel bleiben und nicht ein Haar breit davon weichen, weder zur Rechten noch zur Linken"31, was sich seinerseits auf das Projekt einer Moralisierung des Menschen auswirkt, denn in seinen Augen ist die "philosophische Moral [...] unkräftig, den Menschen zu seiner bestimmten Vollkommenheit zu bringen" ${ }^{32}$. Die Religion stellt für Jung-Stilling also nicht ein Mittel zur Moralisierung, sondern die einzig mögliche Quelle moralischen Verhaltens überhaupt dar.

Die in Jung-Stillings Augen so eindeutigen Karenzen der Vernunft betreffen jedoch nicht nur den Bereich der Erziehung zu moralischem Handeln:

\begin{abstract}
Die Seele hat eine deutliche Vorstellung von gewissen Sachen; sie ist sich deren bewusst, sie ist sich ihres Daseins mit höchster Deutlichkeit bewusst; was ist das nun? Auf, Vernunft! ergründe dich selbst, was du bist! - sie kanns nicht; sie kann nichts weiter entdecken, als dass sie da ist, und dass sie nach gewissen Grundtrieben handelt; sie kann nicht einmal aufhören zu denken, wenn sie will; sie ist also nicht souverain; sie lebt wieder abhänglich von dem, der da befiehlt: $\mathrm{Du}$ Stein sollst ewig fallen, wenn du in den Stand gesetzt wirst, dass du fallen kannst. Es ist also ausgemacht, dass die Vernunft Grenzen um sich herum hat, über welche sie nicht weg kann. Bei diesen Umständen, da die Seele so eingeschränkt ist, hat sie doch einen unendlichen Hunger nach Erkenntnissen. Ein Mensch, der mit diesem Hunger nun in die Dinge der Schöpfung eingeht, stirbt, eh er kaum angefangen hat. [...] Der Mensch ist für dieses Leben nicht allein da, denn er erreicht seinen Endzweck nie ${ }^{33}$.
\end{abstract}

Jung-Stilling zögert offensichtlich, wem er den Wunsch und die Fähigkeit zur Erkenntnis zuschreiben soll: der Vernunft oder der Seele. Wichtiger als dieser Widerspruch ist jedoch die Tatsache, dass er der Vernunft die Erkenntnisfähigkeit eindeutig abschreibt, wodurch die gesamte Existenz des Menschen aus der Immanenz des weltlichen Geschehens in die Transzendenz des göttlichen Willens überführt wird. Die Insuffizienz der Vernunft für das Durchdringen zur absoluten Wahrheit - eine These, aus der der deutsche

29 Vgl. Heinz Dieter KitTSTEINER, "Die Abschaffung des Teufels", in Alexander SCHULLER/ Wolfert VON RAHDEN (Hg.), Die andere Kraft. Zur Renaissance des Bösen, Berlin, Akademie-Verlag, 1993, S. 67.

30 JunG-STILLING, Schleuder eines Hirtenknaben, S. 753, vgl. auch S. 759.

31 Ibid., S. 744.

32 Ibid., S. 722.

33 Ibid., S. 762 
Idealismus erkenntnis- und identitätstheoretische Funken schlagen wird führt bei Jung-Stilling zu einem Kurzschluss: er verbindet den durch die Vernunft nicht zu befriedigenden Wissensdrang eines jeden Individuums mit der doppelten Bestimmung des Menschen, nämlich der moralischen Vervollkommnung und, darauf aufbauend, der Aufnahme in das Reich Gottes. Da beide in der endlichen Welt nicht zu erreichen sind, muss es eine zweite geben. Das Argumentationsschema ist das gleiche wie bei Mendelssohns Beweis der Unsterblichkeit der Seele, nämlich die anthropologische Rückversicherung bei Gott:

\footnotetext{
Ist der Mensch für dieses Leben nicht allein da, so muss ein anderes Leben auf dieses folgen, und ein Mensch muss, seinen wesentlichen Kräften nach, ebenderselbe nach dem Tode sein, der er zuvor war; das ist, er muss sich dieses Lebens noch erinnern können, sonst wäre er so gut, als wenn er neugeboren, das ist, ein anderer Mensch wäre ${ }^{34}$.
}

Auch die Kontinuität von Diesseits und Jenseits verbindet Jung-Stilling mit Mendelssohn, die Akzentuierung ist allerdings höchst unterschiedlich. Insistiert Mendelssohn vor allem auf der Kontinuität der moralischen Verbesserung, so geht es für Jung-Stilling nicht zuletzt auf Grund der Erbsünde um die Möglichkeit der Läuterung, der Einsicht in das vorherige Fehlverhalten. Damit nähert er sich schon der für ihn zentralen Frage, nämlich dem Schicksal des Menschen nach dem physischen Tod. Im Gegensatz zu Mendelssohn verbietet er sich nicht die metaphysische Spekulation, versucht jedoch, diese philosophisch abzusichern. Mittel dazu ist ihm eine eigenwillige Kantlektüre, deren zentrale Bedeutung im autobiographischen Rückblick deutlich gemacht wird:

\begin{abstract}
Kants 'Kritik der reinen Vernunft' las er, natürlicherweise zuerst, [...] Kant beweist da, durch unwiderlegbare Gründe, daß die menschliche Vernunft außer den Grenzen der Sinnenwelt ganz und gar nichts weiß, daß sie in übersinnlichen Dingen, allemal - sooft sie aus ihren eigenen Prinzipien urteilt und schließt - auf Widersprüche stößt, das ist: sich selbst widerspricht; dies Buch ist ein Kommentar über die Worte Pauli: Der natürliche Mensch vernimmt nichts von den Dingen, die des Geistes Gottes sind, sie sind ihm eine Torheit usw.

Jetzt war Stillings Seele wie emporgeflügelt; es war ihm bisher unerträglich gewesen, daß die menschliche Vernunft, dies göttliche Geschenk, das uns von den Tieren unterscheidet, der Religion, die ihm über alles teuer war, so schnurgerade entgegen sein sollte; aber nun fand er alles passend, und gottgeziemend, er fand die Quelle übersinnlicher Wahrheiten in der Offenbarung Gottes an die Menschen, in der Bibel, und die Quelle aller der Wahrheiten, die $\mathrm{zu}$ diesem Erdenleben gehören, in Natur und Vernunft ${ }^{35}$.
\end{abstract}

34 Ibid.

35 Johann Heinrich Jung-Stilling, Lebensgeschichte, [1777-1804], Frankfurt/Main, Insel, 1983, S. 429. 
Jung-Stilling hat Recht und Unrecht zugleich. Einerseits steht er hier, ob er es will oder nicht, auf dem Boden der deutschen Aufklärung, deren Selbstbeschränkung nur allzu deutlich ist. Denn die Frage nach dem Verhältnis von Leben und Tod wird immer noch durch die Religion beantwortet, was nichts anderes bedeutet, als dass die Vernunfterkenntnis innerhalb der von der Religion gesteckten Grenzen bleibt. Andererseits stehen jedoch die Konsequenzen, die Jung-Stilling aus dieser Konstellation zieht, in klarem Widerspruch zu Kants Argumentation. Für Jung-Stilling führen die Denkprinzipien, die in einer den menschlichen Sinnen direkt zugänglichen Welt gelten, in die Irre, sobald sie in dem Bereich Anwendung finden, der darüber hinausgeht. "Das gesamte Gebiet des Transzendenten ist dem direkten Zugriff menschlichen Denkens entzogen, ${ }^{, 36}$. Die philosophisch abgesicherte Begrenzung der Vernunft ist es also, die sich Jung-Stilling bei Kant erschleicht, denn durch die Begrenzung der Vernunft entsteht ein freier Raum, den Kant konsequent für die Wirkung des Moralprinzips benutz ${ }^{37}$, Jung-Stilling jedoch mit Glauben füllt ${ }^{38}$. Damit ist seine Polemik gegen Nicolai zwar rückblickend bestätigt, der durch Kant geschaffene Freiraum wird nun jedoch nicht nur mit Religion, sondern mit den für Jung-Stilling zentralen Spekulationen über das Leben nach dem Tode gefüllt. Konnte er sich in der Spekulation als solcher noch auf Aufklärer wie Mendelssohn beziehen, so wird in der theoretischen Begründung seiner weiteren Vorgehensweise der Bruch mit der Aufklärung eindeutig, denn für Kant stellt die Seele nur ein Gedankenkonstrukt dar, das die Vernunft aus sich selbst erzeugt, weshalb der Verstand von ihr nichts erkennen kann. Aussagen über die Seele sind nicht möglich, denn sie sind weder beweisbar noch nachvollziehbar und gehören daher in den Bereich der spekulativen Vernunft, mit anderen Worten des Glaubens. Gerade das will Jung-Stilling jedoch nicht akzeptieren, denn es geht ihm darum, seine Spekulationen zu beweisen und als Wahrheiten zu verbreiten. Möglich ist das allerdings nur durch die radikale Umkehrung von Vernunft und Unvernunft; Ausgangspunkt dabei ist die Infragestellung jeglicher naturwissenschaftlicher Erkenntnis:

\footnotetext{
Alle Vorstellungen, die sich auf Raum und Zeit gründen, sind eingeschränkt; da nun Gott der Ewige, Unendliche und Unbegreifliche keine Schranken kennt, so stellt er sich auch die Welt nicht in Raum und Zeit vor; da nun seine Vorstellungen allein Wahrheit sind, so ist auch die Welt nicht in Raum und Zeit; ferner: da das, was wir Körper und Materie nennen, einen Raum einnimmt, durch die Zeit fortdauert, und die Dinge außereinander sich im Raum bewegen, durch Kräfte aufeinander wirken usw., Raum und Zeit aber wörtlich in der Schöpfung nicht existieren, sondern nur Vorstellungsformen sind, so ist das, was
}

36 VINKE, Jung-Stilling und die Aufklärung, S.316. Zu Jung-Stillings Briefwechsel mit Kant und dem auch dort deutlich werdenden strategischen Nichtverstehen der vorsichtigen Hinweise Kants vgl. Ibid., S. 318.

37 Vgl. den vierten Teil der Religion in den Grenzen der blossen Vernunft, Stuttgart, Reclam, 1981.

38 VINKE, Jung-Stilling und die Aufklärung, S. 319. 
wir Materie, Kraft und Wechselwirkung aufeinander nennen, blos menschliche Vorstellung, in der Wahrheit befindet sich alles anders ${ }^{39}$.

In Jung-Stillings Augen ist das kopernikanische System nur eine Vorstellung unter anderen, denn es ist "durch Vernunftschlüsse" entstanden, die sich auf "die Wirklichkeit des Raums und der Zeit gründen, und also nicht wahr sind" 40 . Anders gesagt bedeutet für ihn die rational-physikalische Erkenntnis ein rein menschliches Wissen und in dem Sinne einen unverbindlichen und hilflosen Orientierungsversuch, der keinerlei Wahrheit für sich beanspruchen kann, wodurch naturwissenschaftliche Argumente der Neologen gegen biblische Wahrheiten und religiöse Spekulation jegliche Legitimität verlieren. Umgekehrt - und hier liegt der Umschlag von Vernunft in Unvernunft - versteht Jung-Stilling seine eigenen Spekulationen über das Leben nach dem Tode - und insbesondere den Hades als Zwischenraum zwischen Erde und Reich Gottes - als religiös fundiertes Erfahrungswissen, das als solches Evidenz besitzt und allgemeinen Geltungsanspruch besitzt.

\section{Jung-Stilling und die Geister}

Der Kampf gegen die Aufklärungstheologie stellt jedoch nur eine von Jungs-Stillings Triebfedern dar. Gleichermaßen grundlegend für seine Argumentation in der Geisterkunde ist die durch die französische Revolution ausgelöste Endzeiterwartung. Das Ende des Ancien Régime bedeutet für ihn den Höhepunkt einer kollektiven Entfernung von Gott, die mit der kopernikanischen Wende in den Naturwissenschaften eingesetzt hatte. Er versteht diese als einen ersten nachhaltigen Angriff auf das christliche Weltbild, auf den dann die Trennung der Philosophie von der Theologie gefolgt sei, die ihrerseits mit den im 18. Jahrhundert entwickelten sogenannten "mechanischen" Denksystemen zwangsläufig zur Verbreitung des Atheismus geführt habe ${ }^{41}$.

Jung-Stilling geht also von einer Kontinuität der Aufklärung bis in seine Gegenwart aus und versteht generell seine Gegenwart auf Grund der sie prägenden Säkularisierungstendenzen als Zeitalter der Aufklärung. Und diese wurde im Wesentlichen von protestantischen Denkern vorangetrieben, so dass sich Jung-Stilling, zu einer vorsichtigen und für seine soziale Stellung nicht ungefährlichen Annäherung an den Katholizismus gezwungen sieht ${ }^{42}$. Denn

39 Johann Heinrich Jung-STILling, Theorie der Geisterkunde, Nürnberg, 1808, ND Wiesbaden, Fourier, 1979, S. 33.

40 Ibid., S. 35.

41 Ibid., S. 24.

42 Er unterscheidet in seiner Gegenwart drei verschiedene Bewusstseinsformen: die Katholiken, die weiterhin an die Existenz der Geister glauben, während die protestantisch sozialisierten Bürger sich in zwei Gruppen aufspalten, einerseits diejenigen, die die Offenbarung auf Moral reduzieren, andererseits die dominierende Mehrheit, die weder an Gott noch an die Geisterwelt glauben. Vgl. Ibid., S. 3-5. Zur vorsichtigen Kritik Jung-Stillings an den 
zur Zeit von Newton und Descartes hatten allein der Papst und die katholische Kirche die drohende Gefahr verstanden, waren aber unfähig gewesen, diese effizient einzudämmen. Die Folge war eine Wechselwirkung vor allem deutscher und französischer religionsfeindlicher Denker - zunächst Voltaire und Rousseau, dann die Neologen - deren subversive Tätigkeit sich auch in der Politik niederschlug und in der Revolution einen vorläufigen Höhepunkt fand, der jedoch nun eine für die Mehrheit der Bevölkerung unerwartete Auswirkung haben wird:

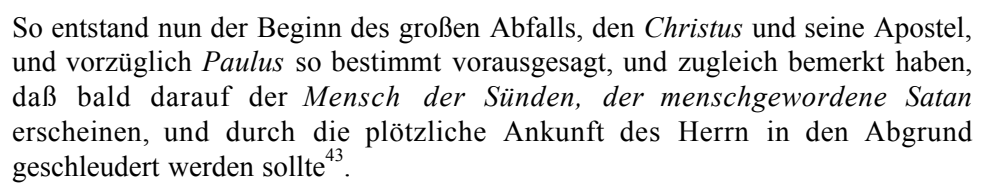
und vorzüglich Paulus so bestimmt vorausgesagt, und zugleich bemerkt haben, daß bald darauf der Mensch der Sünden, der menschgewordene Satan erscheinen, und durch die plötzliche Ankunft des Herrn in den Abgrund geschleudert werden sollte ${ }^{43}$.

Geht die "Aufklärung" zu Beginn des 19. Jahrhunderts nun "dem ewigen Verderben entgegen" 44 , so bedeutet dies nichts anderes als den Endkampf zwischen Gut und Böse. Und gerade dafür müssen die Menschen laut JungStilling vorbereitet werden. Und daher ist Aufklärung notwendig: Aufklärung über die Irrtümer und die Aporien der historischen Aufklärung. Zentraler Ansatzpunkt ist dabei in Jung-Stillings Augen der Kampf gegen die Postulate der Neologen: die Wunder Jesu wie die Existenz von Geistern und Gespenstern müssen wieder in ihrer metaphysischen Evidenz deutlich gemacht werden. Denn die Revision der neologischen Postulate bedeutet nichts anderes als Einstimmung und Vorbereitung auf das tatsächliche Reich Gottes. Und zu dem gehören auch die Geister:

\footnotetext{
Dass gute und böse Engel und Geister mächtig auf uns und die Sinnenwelt wirken, das behauptet die Bibel, und weder die Vernunft, noch die Natur haben etwas dagegen einzuwenden, im Gegenteil, der aufmerksame Beobachter findet zu Zeiten unleugbare Spuren solcher Einwirkungen, wie sich im Verfolg zeigen wird. [...] Unsere körperliche physische Natur ist in unserem gegenwärtigen Zustand blos auf unsere Sinnenwelt organisiert und eingerichtet; in unserem natürlichen Zustand empfinden wir ausser unserer eigenen Seelen, von der Geisterwelt nichts; und da auch unsere Vernunft nur auf sinnliche Erfahrungen ihre Schlüsse gründen kann, so weiss sie aus sich selbst, und aus eigenen Quellen ebenso wenig von einer Geisterwelt und ihren Wirkungen. Nur die göttliche Offenbarung, und dann von jeher einzelne Erfahrungen, belehren uns, dass sich Wesen aus der Geisterwelt, und auch Gott selbst sinnlich gezeigt haben, und auf unsere Sinnenwelt wirken ${ }^{45}$.
}

Reformatoren insbesondere hinsichtlich der Abschaffung des Fegefeuers vgl. Ibid., S. 16 und JUNG-STILLING, Apologie der Geisterkunde, S. 29.

43 Jung-STILLING, Lebensgeschichte, S. 460. Wenige Seiten weiter wird er seine Postulate explizit an den Chiliasmus von Johann Albrecht Bengel binden. Vgl. Ibid., S. 490.

44 Jung-STILLING, Theorie der Geisterkunde, S. 23.

45 Ibid., S. 41-42. 
Jung-Stillings Vorgehensweise ist strategisch-kohärent und vollkommen willkürlich zugleich. Die Welt der Geister ist seiner Argumentation zufolge der Vernunft nicht zugänglich, also kann sie von dieser auch nicht dementiert werden, so dass zunächst die Bibelauslegung als einzige Quelle bzw. Beweis übrig bleibt. Denn erst nach seinem physischen Tod soll der Mensch zur Erfahrung des Übersinnlichen - in Jung-Stillings Worten des außerhalb von Raum und Zeit befindlich Göttlich-Geistigen - in der Lage sein. Mit dieser These hätte Jung-Stilling seinen eigenen Spekulationen den ohnehin schon dünnen Boden entzogen, gäbe es nicht die im obigen Zitat angesprochenen Zeugenaussagen über seltene, aber immer wieder vorkommende physische Offenbarungen Gottes und der Geister, auf die sich Jung-Stilling in der Geisterkunde permanent bezieht. Und auch hier ist er nur konsequent, wenn er notiert, die betroffenen Personen, also die Geisterseher, hätten die Erscheinungen mit allen ihren "Sinnen geprüft" und wenn er daraus schließt, er verfüge daher über "Fakta",", die als solche anzuerkennen seien. Anders gesagt bleibt Jung-Stilling auf dem Boden des für seine Vorgehensweise charakteristischen tautologischen "autobiographischen Gottesbeweises" Sein Glaubensbekenntnis und sein "religiöses Lehrsystem" 48 besitzen allgemeinen Wert, da sie durch seine Interpretation seines Lebenswegs bewiesen werden ${ }^{49}$. Genau dieser Mechanismus wird nun auf die ebenfalls autobiographischen "Zeugenaussagen" übertragen, die Jung-Stilling als Beweise für göttliche Offenbarungen versteh $t^{50}$ und aus denen er dann seine Geisterwelt konstituiert.

Die Argumentation hat also ihre eigene Kohärenz und verbietet zugleich jede Debatte, da es sich um Wahrnehmungen handelt, die zwar wie die Interpretation der entsprechenden Bibelstellen allein auf der Subjektivität des Autors beruhen, sich aber wegen der Trennung von Vernunft und Transzendenz jedem Einwand entziehen. So bleibt dem Leser nur der Versuch der Rekonstruktion, wobei zunächst deutlich wird, dass es JungStilling eindeutig um die Aufhebung der Trennung von Diesseits und Jenseits geht $^{51}$. Um diese angebliche Kontinuität zu beweisen, lässt er seiner Phantasie freien Lauf, schöpft im Arsenal vorchristlicher Weltbilder, die er je nach Bedarf umgestaltet, sowie, wie gesagt, im unerschöpflichen Fundus der Legenden und Anekdoten. So berichtet er z.B. von einem Freund, der ihn über das Ahnungsvermögen einer Frau informiert hat; da Jung-Stilling die

46 Jung-STILling, Apologie der Geisterkunde, S. 53.

47 Theologische Realenzyklopädie, Berlin - New York, De Gruyter, 1988, Bd. XVIII, S. 467.

48 JUNG-STILLING, Lebensgeschichte, S. 586.

49 JunG-STILLING, Lebensgeschichte, S. 570.

50 JunG-STILLING, Theorie der Geisterkunde, S. 189.

51 Dabei nimmt die Semantik oftmals ungewollt karikaturale Formen an. Etwa wenn JungStilling den Hades zunächst relativ neutral als "Verbesserungs- und Reinigungsort", dann jedoch als eines der "Zucht- und Verbesserungshäuser" bezeichnet, in denen die Menschen für ihre Fehler haften müssen. JUNG-STILLING, Apologie der Geisterkunde, S. 50 und S. 56. 
"Aufrichtigkeit" 52 der betroffenen Personen bestätigen kann, erhalten deren Behauptungen sofort einen für ihn unwiderlegbaren Wahrheitscharakter. Andere Beispiele sind natürlich das Carolinum in Braunschweig, das Magazin für Erfahrungs-Seelenkunde und vor allem das Museum des Wundervollen $^{53}$, deren Berichte über Geistererscheinungen für ihn Erfahrungswahrheiten darstellen:

$\mathrm{Da}$ alle Erfahrungen die von jeher bis dahin in Ansehung des Geisterreiches gemacht und beobachtet wurden - insofern sie von Phantasien und Schwärmereien entfernt sind - sich genau an die göttliche Offenbarung anschließen und gleichsam fortgesetzte Offenbarungen sind, so bestätigt das Eine die Wahrheit des Anderen, folglich auch die Wahrheit der christlichen Religion nach dem alten apostolischen System ${ }^{54}$.

Er sammelt "von verschiedenen Orten her, Nachrichten von Erscheinungen aus dem Geisterreich; vom Wiederkommen längst und vor kurzem verstorbener Personen hohen und niedern Standes"55, die er in seinem Sinne interpretiert und zugleich als unwiderlegbare Beweise vorstellt:

\begin{abstract}
Stillings Begriffe vom Hades, von der Geisterwelt, vom Zustand der Seele nach dem Tode, sind nächst denen, in der heiligen Schrift zum Nachdenken hingeworfenen Winken, aus diesen Quellen geschöpft; indessen sind das keine Glaubensartikel, jeder mag davon halten was er will; nur dass er sie nicht verurteile: denn dadurch würde er sich zugleich selbst verurteilen ${ }^{56}$.
\end{abstract}

Doch es bleibt nicht bei diesen willkürlichen Interpretationen, denn JungStilling schreckt nicht vor der reinen Spekulation zurück, wie es bei dem Rückgriff auf den Äther als Verbindungsmittel zwischen Menschenwelt und Geisterwelt ${ }^{57}$ deutlich wird. Zunächst formuliert er die für ihn evidente Notwendigkeit eines Kontaktes von Diesseits und Jenseits:

Es ist durchaus nötig, dass der ewige, von Gott ausgegangene vernünftige Geist des Menschen, ein Organ haben muss, wodurch er auf andere Wesen, und diese wieder auf ihn zurückwirken können; ohne dies könnte er ja von nichts außer sich Erkenntnis haben, und er selbst wäre für alle andere Wesen ein pures Unding. Dieses Organ nun ist der Äther, der durch keine Naturkraft zerstörbar, sondern ewig und unveränderlich ist ${ }^{58}$.

52 JUNG-STILLING, Theorie der Geisterkunde, S.154.

53 Die Zeitschrift wurde zwischen 1803 und 1811 von Johann Adam Bergk und Friedrich Gotthilf Baumgärten in Leipzig veröffentlicht. Vgl. Karl Riha (Hg.), Museum des Wundervollen oder Magazin des Ausserordentlichen - Geschichten aus dem phantastischen Untergrund - ein anregendes Archiv für den Beobachter des täglichen $u$. nächtlichen Lebens, ND Köln, Diederichs, 1984.

54 JunG-STILling, Theorie der Geisterkunde, S. 189.

55 JunG-STILLING, Lebensgeschichte, S. 481.

56 Ibid., S. 482.

57 Zur Rolle des Äthers in vorchristlichen Weltbildern vgl. Dieter HARMENING, Wörterbuch des Aberglaubens, Stuttgart, Reclam, 2009, S. 52.

58 JunG-STILLING, Theorie der Geisterkunde, S. 64. 
Es folgen Anekdoten und Gerüchte, in Jung-Stillings Augen unwiderlegbare Zeugenaussagen, denen er den seine eigenen Schlüsse zieht, denen er dann den Status von Erfahrungswahrheiten zuspricht:

Es ist also eine unstreitige Erfahrungswahrheit, dass in dem menschlichen Körper ein feines Lichtwesen, eine ätherische Hülle des unsterblichen vernünftigen Geistes sei, welche sich im Magnetismus, im Galvanismus, in der Elektrizität, und in Sympathie und Antipathie unwidersprechlich darstellt, und auf mancherlei Weise wirksam zeigt; hiermit ist der vernünftige Geist ewig und unzertrennlich verbunden. Ich nannte diesen inneren Lichtmenschen oben die Menschenseele ${ }^{59}$.

Der Vorwurf des Aberglaubens oder der Spekulation wird dabei kategorisch zurückgewiesen:

Endlich bitte ich $\mathrm{zu}$ bedenken, ob die wirkliche Erscheinung eines abgeschiedenen Geistes, ohne mein Mitwirken - Aberglauben genannt werden könne? - ist das Aberglauben, wenn ich bei hellem Bewusstsein, einen Irrwisch, oder sonst eine seltene Naturerscheinung sehe? - es kommt hier nur darauf an, was ich für einen Gebrauch davon mache? - ich werde also auch zeigen, wie man sich bei solchen Erscheinungen vernünftig und christlich zu verhalten habe $^{60}$.

Geister existieren somit für Jung-Stilling in verschiedenen Arten, "die immer dem Grade der Vollkommenheit nach, von einander verschieden sind, aber doch alle miteinander umgehen, und auf einander wirken"61, und die auch auf die Lebenden Einfluss nehmen können. Soweit das Postulat. Daher bedarf es eines Organs, um den Kontakt herzustellen; es ist "nötig", also muss es existieren. Schon in seiner Autobiographie ${ }^{62}$ hatte sich Jung-Stilling auf

59 Ibid., S. 78

60 Ibid., S. 222.

61 Ibid., S. 38.

62 "Der Gemütszustand, in welchen Stilling während dem Ausarbeiten dieses, vier große Oktavbände starken, Buchs versetzt wurde, ist schlechterdings unbeschreiblich; sein Geist war wie in ätherische Kreise emporgehoben; ihn durchwehte ein Geist der Ruhe und des Friedens, und er genoß eine Wonne, die mit Worten nicht beschrieben werden kann. Wenn er anfing zu arbeiten, so strahlten Ideen seiner Seele vorüber, die ihn so belebten, daß er kaum so schnell schreiben konnte, als es der Ideengang erforderte; daher kam es auch, daß das ganze Werk eine ganz andere Gestalt, und die Dichtung eine ganz andere Tendenz bekam, als er sie sich im Anfang gedacht hatte.

Hierzu kam nun noch eine sonderbare Erscheinung: in dem Zustande zwischen Schlafen und Wachen stellten sich seinem innern [sic] Sinn ganz überirdisch schöne, gleichsam paradiesische Landschaftsaussichten vor - er versuchte sie zu zeichnen, aber das war unmöglich. Mit dieser Vorstellung war dann allemal ein Gefühl verbunden, gegen welches alle sinnliche Vergnügungen wie nichts zu achten sind - es war eine selige Zeit! - dieser Zustand dauerte genauso lang, als Stilling am Heimweh schrieb, nämlich vom August 1793 bis in den Dezember 1794, also volle fünfviertel Jahr.

Hier muß ich aber den christlichen Leser ernstlich bitten, ja nicht so lieblos zu urteilen, als ob Stilling sich dadurch etwa einer göttlichen Eingebung, oder nur etwas Ähnliches, anmaßen wolle. - Nein Freunde! Stilling maßt sich überhaupt gar nichts an: - es war eine erhöhte Empfindung der Nähe des Herrn, der der Geist ist; dies Licht strahlte in seine Seelenkräfte, und 
den Äther bezogen, um eine mystische Erfahrung während des Schreibprozesses wiederzugeben. Dabei hatte es sich jedoch noch um eine weitgehend metaphorische Verwendung des Begriffs gehandelt, während es nun um die definitive Aufhebung der Trennung von Diesseits und Jenseits geht. Wie Lavater, der ebenfalls über den Äther spekuliert und der allerdings behauptet, der Mensch bekomme erst in einem Zwischenraum nach dem Tode einen "ätherischen Lichtleib", geht es Jung-Stilling eindeutig um eine dauerhafte und unmittelbare sensitive Transzendenzerfahrung im Diesseits ${ }^{63}$.

Dank der Verbindung zu den Geistern ist diese Manifestation der Transzendenz in der Immanenz also möglich. Nun stellt sich abschließend die Frage nach der Lokalisierung der Geister. Laut Jung-Stilling befinden sie sich im Hades, der sich von der Erdmitte bis in die höhere Atmosphäre erstreckt, wo er an den Himmel grenzt. Er ist der Aufenthaltsort abgeschiedener Seelen, guter und böser Engel, und verschiedenster Geister. Laut Jung-Stilling gelangen allein die Heiligen und die Menschen, die ein wahrhaft tugendhaftes Leben geführt hatten gleich nach ihrem Tod in den Himmel, während die Mehrheit der Personen erst das Zwischenstadium des Hades durchlaufen muss. Es handelt sich bei ihnen um die Seelen von Menschen, die "noch Irrdischgesinntheit, Lieblingsneigungen, und Leidenschaften" ${ }^{, 64}$ aufweisen. Sie erfahren im Hades eine Läuterung, die von unterschiedlicher Dauer sein kann. Auf dem Spiel steht die Loslösung von jeglicher Orientierung am Diesseits. Deshalb müssen sie ihre "Einbildungskraft von allen den Bildern reinigen, und nach und nach so wie diese Bilder abnehmen, auch die Liebe zum Irdischen verlieren"65. Durch "Entbehrung alles dessen was ihnen lieb ist, und jeden Genusses, und dann durch das traurigste Heimweh nach dem verschwundenen Erdenleben" gelangen",67.

Auf die Nähe dieser Spekulationen zum katholischen Verständnis des Fegefeuers kommen wir später zu sprechen. Festzuhalten ist zunächst, dass für Jung-Stilling der Aufenthalt der Seelen im Hades in schweren Fällen mehrere Jahrhunderte dauern kann $^{68}$, dass er aber nicht unbedingt permanent

erleuchtete die Imagination und die Vernunft. In diesem Licht sollte Stilling das Heimweh schreiben; aber deswegen ist es doch immer ein gebrechliches Menschenwerk: wenn man einem Lehrjungen, der bisher beim trüben Licht armselige Sachen machte, auf einmal die Fensterladen öffnet, und die Sonne auf seine Werkstätte strahlen läßt, so macht er noch immer Lehrjungenarbeit, aber sie wird doch besser als vorher", JUNG-STILLING, Lebensgeschichte, S. 468-469.

$63 \mathrm{Zu}$ Lavater vgl. Horst WeIGeLt, Johann Kaspar Lavater. Leben, Werk und Wirkung, Göttingen, Vandenhoeck \& Ruprecht, 1991, S. $74 f$.

64 JunG-STILLING, Theorie der Geisterkunde, S. 264.

65 Ibid., S. 297.

66 Ibid., S. 296.

67 Ibid., S. 298.

68 Ibid., S. 48. Damit nähert sich Jung-Stilling dem Fall Lavater, der in einem Brief an Carl Heinrich von Gleichen diesem mitteilte, er werde dem Apostel Johannes, von dessen Weiterleben auf Erden er überzeugt war, ein Zimmer zur Verfügung stellen, in dem dieser diskret übernachten könne, um so mit ihm, Lavater, in Ruhe diskutieren zu können. Vgl. 
ist. Eine kurzfristige Rückkehr ins Diesseits ist den Seelen ebenso möglich wie der Kontakt zu den Lebenden und der Einfluss auf deren Leben. Gerade das erklärt in Jung-Stillings Augen, warum das Leben des Menschen einer vielfachen Heteronomie unterworfen ist. Und erst dann, wenn der Mensch sich dieser Vielfalt von Abhängigkeiten - aber damit auch der direkten Verbindung von Immanenz und Transzendenz - bewusst ist, kann er sein Leben wahrhaft christlich gestalten. Für Jung-Stilling ist damit der Kreis geschlossen:

\begin{abstract}
Die Ahnungen, Gesichte, und Geistererscheinungen zeugen von einer unsichtbaren Geisterwelt, die der Aufenthalt abgeschiedener Seelen, guter und böser Engel, und Geister ist; sie beweisen die Fortdauer unserer Seelen nach dem Tod, mit dem klaren Bewusstsein ihrer gegenwärtigen Existenz, und der Rückerinnerung des ganzen vergangenen Erdenlebens; dann auch die große Wahrheit von Belohnungen und Strafen nach diesem Leben ${ }^{69}$.
\end{abstract}

\title{
Jung-Stilling und das Erbe der Aufklärung um 1810
}

Schaut man auf Jung-Stillings Argumentation und ihre Affinitäten mit Lavater zurück, so steht eins fest: ein Fall wie Lavater würde heutzutage mit Sicherheit in der Psychiatrie landen und diese so schnell nicht verlassen. Und auch Jung-Stilling würde einer genauen Untersuchung unterzogen werden. Warum? Nicht zuletzt auf Grund der Durchsetzung des modernen Wissens, so wie sie sich zu Beginn des 19. Jahrhunderts abzeichnet. Als Beleg dafür können die Definitionen der Begriffe Geist und Gespenster gelten, so wie sie zum Zeitpunkt der Veröffentlichung der Geisterkunde von Adelung und Campe vorgeschlagen werden. Dabei ist es fast unnötig zu betonen, dass beide wie selbstverständlich von der Unsterblichkeit der menschlichen Seele ausgehen $^{70}$, während in Hinblick auf übersinnliche Wesen die für eine Übergangsperiode charakteristische Vorsicht deutlich wird. So bestätigt Adelung die Existenz der Geister nachdrücklich ${ }^{71}$ und stellt sie sogar durch den Verweis auf die "Weltweisheit" in einen direkten Bezug zur menschlichen Praxis: die "Geisterlehre" ist "die Lehre von den Kräften der

Diethard SAWICKI, "Die Gespenster und ihr Ancien Régime: Geisterglauben als 'Nachtseite' der Spätaufklärung", in Monika NEUGEBAUER-WÖLK (Hg.), Aufklärung und Esoterik, Enlightenment and Esotericism. Reception - Integration - Confrontation, Tübingen, Niemeyer, 2009, S. 364ff.

69 JunG-STILLING, Theorie der Geisterkunde, S. 9.

70 Artikel unsterblich, in Johann Christoph ADELUNG, Grammatisch-kritisches Wörterbuch der Hochdeutschen Mundart, Ausgabe Leipzig, 1811, Bd. IV, S. 893, und Wörterbuch der deutschen Sprache, veranstaltet und herausgegeben von Joachim Heinrich Campe, Bd. 5, Braunschweig, 1811, S. 188.

71 "Die guten Geister, die guten Engel, zum Unterschiede von den bösen, oder Teufeln. Im gemeinen Leben drückt man mit dem Worte Geist oft ein solches Wesen höherer Art aus, ohne eben zu bestimmen, ob es zu den guten oder bösen Geistern gehöre. Es lässt sich ein Geist sehen, ein Gespenst. Es ist ihm ein Geist erschienen", Artikel Geist, ibid., Bd. 2, S. 515. 
Geister, Pneumatologia, welche einen Theil der Weltweisheit ausmacht; ingleichen ein Buch, worin diese Lehre abgehandelt worden",72. Welche Form dieser Praxisbezug annehmen soll, bleibt jedoch offen, dies umso mehr als dass ein "Geisterseher" eindeutig als "ein Schwärmer, welcher Geister zu sehen glaubt" ${ }^{, 73}$ abqualifiziert wird und die Existenz von Gespenstern durch die Einbildungskraft des Menschen erklärt wird ${ }^{74}$. Campe seinerseits bewahrt hinsichtlich der Geister eine größere Distanz, wenn er betont, dass das Wissen über Geister keinen Wahrheitsstatus für sich in Anspruch nehmen kann:

[...] überhaupt ein einfaches, unsichtbares Wesen, dem Kraft und Leben zugeschrieben wird [...]. In engerer Bedeutung, ein jedes einfaches, unkörperliches Wesen, welches die Kraft zu denken und zu wollen hat. So werden die Engel überhaupt Geister genannt, und man unterscheidet die guten Geister, die guten Engel, von den bösen Geistern, den gefallenen Engeln oder Teufeln. Die Geister der Verstorbenen. [...] Im gemeinen Leben wird das Wort Geist von einem höhern Wesen, ohne zu bestimmen, ob es ein gutes oder böses gemeint sei oder auch von dem Geiste eines Verstorbenen, der den Augen sichtbar erscheinen soll, für Gespenst, für Abgeschiedene gebraucht ${ }^{75}$.

Es handelt sich also nur um Vermutungen, die Eigenschaften werden den Wesen "zugeschrieben". Wird auch hier noch Vorsicht deutlich, so herrscht in Bezug auf die Gespenster Eindeutigkeit, da deren Existenz explizit dem Aberglauben zugeschrieben wird:

Ein unvermutet und schrecklich erscheinendes Wesen, Ding, das nach der Meinung abergläubiger Leute ein Geist, im Grunde aber nichts als ein Gebilde ihrer durch Furchtsamkeit in Bewegung gesetzten Einbildungskraft ist oder ein verkappter Mensch, der zu Schrecken oder sonst eine Absicht zu erreichen sucht $^{76}$.

Jung-Stillings Spekulationen stehen also quer zu dem Wissenswandel seiner Zeit. Und doch wäre es verfehlt, ihn ganz von der Aufklärung lösen zu wollen, denn auch bei ihm wird an manchen Stellen eine bezeichnende Widersprüchlichkeit deutlich. So notiert er über die Existenz des Teufels:

Dass also böse Menschen entweder durch natürliche Disposition oder auch durch Kunst, ihr Ahnungsvermögen entwickeln und so auch mit bösen Geistern in Verbindung kommen können, das ist keinem Zweifel unterworfen; ob aber die bösen Geister noch die Macht haben, die ihnen der Aberglaube zuschreibt, das ist eine andere Frage. Seit dem Sieg und Triumph unseres hochgelobten Erlösers hört ihr Despotismus, ihre Zwangsherrschaft über die Menschen auf; nur

72 Ibid.

73 Ibid.

74 Ein Gespenst ist "eine geistige Substanz, wenn sie unter einer angenommenen Gestalt den Menschen erscheinet; doch am häufigsten nur von solchen Substanzen, welche, wie man sich einbildet, den Menschen nur zur Plage, oder zum Schrecken erscheinen", Artikel Gespenst, ibid., S. 631.

75 Artikel Geist, in Wörterbuch der deutschen Sprache, veranstaltet und herausgegeben von Joachim Heinrich Campe, Bd. II, Braunschweig 1808, S. 278.

76 Artikel Gespenst, ibid., Bd. II, S. 345. 
diejenigen, die sich freiwillig von ihnen verführen und leiten lassen, die beherrschen sie; böse, gottlose Menschen sind in ihrer Gewalt, und doch auch nur so lange als sie selbst wollen ${ }^{77}$.

Die Reflexion ist in mehrerer Hinsicht bemerkenswert, denn Jung-Stilling nähert sich zunächst den Positionen aufgeklärter Theologen wie Semler, wenn er auf den durch das Christentum angeblich vollzogenen Bruch hinweist ${ }^{78}$ und den freien Willen des Menschen betont. Im gleichen Atemzug betont er jedoch, dass bewusst unmoralisch handelnde Menschen in die Gewalt des Teufels kommen können, wodurch der Schritt zurück zur Orthodoxie vorbereitet wird:

Dann kämpfen auch die bösen Geister mit allerhand Waffen gegen wahre Christen $[. .$.$] aber sie können nie siegen, oder der Mensch ist selbst schuld$ daran. Widerstehet dem Teufel, so fleucht er von euch - Nur in den Kindern des Unglaubens treibt er sein Wesen, da übt er noch seine Gewalt aus ${ }^{79}$.

Damit werden sowohl die physische Existenz des Teufels als auch die Möglichkeit eines Bündnisses mit dem Menschen wieder eingeführt ${ }^{80}$. Gleiches gilt für die Hexen, in deren Fall Jung-Stilling ebenfalls versucht, eine mediane Position einzunehmen, tatsächlich jedoch am voraufgeklärten Weltbild festhält:

Was man von Behexen, von allerhand Krankheiten, und koerperlichen Uebeln an Menschen und Vieh zu glauben pflegt, und der Hexerey zuschreibt, das ist Aberglaube, und gewoehnlich entweder Betrug, und Täuschung, oder eine Krankheit, ein Umstand, den die Aerzte noch nicht aus natürlichen Ursachen erklären können. Seitdem Jesus Christus auf dem Thron der höchsten Majestät zur Rechten des Vaters sitzt, hat der Satan keine Macht mehr über den Menschen

[...]. Hexen und Zauberer können also durch ihre Verbindung mit bösen

77 Jung-STILLING, Theorie der Geisterkunde, S. 190.

78 "Der Teufel [...] ist blos in moralischem geistlichen Verstande und Umfange Teufel; er war nie Herr oder Mitherr über die göttlichen Gesetze der Natur. Irrtümer, Unwissenheit und geistlichen Tod befördert dieser Teufel und Mörder der Menschen vom Anfange an ; Christi Erlösung ist an sich auch geistlich, und kann nur im Geist, geistlich, durch unsere lebendige Erkenntnis und neue Fertigkeit des Verstandes und Willens, angenommen und genützt werden". Johann Salomo SEMLER, Sammlungen von Briefen und Aufsätzen über die Gassnerischen und Schröpferischen Geisterbeschwörungen, Halle, Carl Hermann Hemmerde, 1776, Bd. II, S. XXVIII. Gerade was Schröpfer betrifft, ist Jung-Stillings Position von größter Ambivalenz, wie in folgender Reflexion deutlich wird: "Die Geschichte, die ich aus Eckartshausens Buch [Karl von Eckartshausen, Aufschlüsse zur Magie aus geprüften Erfahrungen über verborgene philosophische Wissenschaften und verdeckte Geheimnisse der Natur. München, Joseph Lentner 1791-1795] erzähle, wo man durch lange Vorbereitung seine Imagination erhitzt, und dann durch einen gefährlichen narkotischen Dampf so betäubt wird, dass man das verlangte Bild im Dampf sieht, beweist ja nicht, dass ich die Möglichkeit des Geisterzitierens glaube, sondern dass bei diesem Kunststück Dinge vorkommen, die unbegreiflich sind, und doch einen fernen Einfluss des Geisterreiches ahnen lassen. Wer Schröpfern gekannt, und seine Betrügereien gehörig beobachtet hat, der wird mir recht geben”, JUNG-STILLING, Apologie der Geisterkunde, S. 50-51.

79 Jung-STILling, Theorie der Geisterkunde, S. 190.

80 Ibid., S. 138. 
Geistern, niemand schaden, wohl aber, so wie jeder andere böse Mensch, durch Beibringung eines Gifts, oder sonst einer schädlichen Sache ${ }^{81}$.

Hexen und Zauberer können in Verbindung mit bösen Geistern niemandem schaden - zumindest nicht mehr als böse Menschen. Damit sind sie aber wieder da! Gerade deshalb erscheint es sehr diskutabel, wenn man Jung-Stillings Spekulationen als "Abschluss und Synthese der aufgeklärten Reflexion über die Geister" bezeichnet ${ }^{82}$. Dies ist allein auf Grund der in der Geisterkunde benutzten "Methode" unmöglich. Denn selbst wenn man konzediert, dass auf Grund des Dogmas der Unsterblichkeit der Seele die Existenz von Geistern auf dem epistemologischen Boden der deutschen Aufklärung nicht bestritten werden kann, so handelt es sich doch bei JungStilling nicht um empirisch abgesicherte Reflexionen, wie gerade an seinem Erfahrungsbegriff deutlich wird. Damit steht und fällt jedoch die Einordnung seines Werks:

\footnotetext{
Diese übersinnlichen Erscheinungen werden von Jung-Stilling nicht wie Gegenstände des Glaubens akzeptiert, sondern als natürliche und kritisch nachprüfbare Erfahrung behandelt, die vernunftmäßig zu beweisen ist ${ }^{83}$.
}

Bei Jung-Stilling findet man nur extravagante Spekulationen - sei es über das drohende Weltende, die Existenz von Hexen und Gespenstern oder die dem Menschen zur Verfügung stehenden Organe zum Kontakt mit dem Jenseits. In allen diesen Fällen übernimmt er kolportierten Anekdoten - in der Tat vor allem von Mathematikern und anderen "Rationalisten" -, denen er dann den Status von Erfahrungswahrheiten zuschreibt. Gerade das von JungsStilling beanspruchte Postulat der nachprüfbaren Erfahrung erweist sich damit als unhaltbar und man könnte an die für Empiriker notwendige Bescheidenheit erinnern:

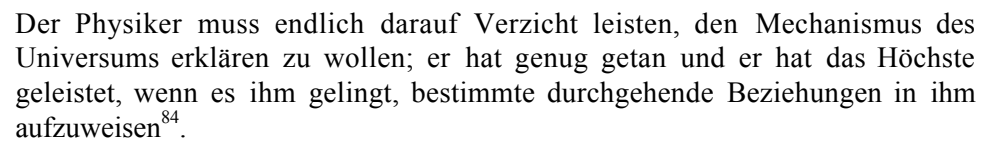
Universums erklären zu wollen; er hat genug getan und er hat das Höchste geleistet, wenn es ihm gelingt, bestimmte durchgehende Beziehungen in ihm aufzuweisen $^{84}$.

So Cassirer in Bezug auf Condillac; gerade diese Beziehungen fehlen jedoch bei Jung-Stilling und Gleiches gilt für die Nachprüfbarkeit der angeblichen Fakten. Bei ihm gibt es bestenfalls die Nachglaubbarkeit.

Ganz anders steht es jedoch um seine Spekulationen über den Hades, in denen tatsächlich Versatzstücke aufklärerischen Denkens zum Ausdruck kommen und die Widersprüchlichkeit von Umbruchperioden wie den Jahren um 1810 deutlich machen. Die Geisterkunde wurde bekanntlich auf Grund

81 Ibid., S. 191.

82 Anne CONRAD, “'Umschwebende Geister' und aufgeklärter Alltag. Esoterik als Religiosität der Spätaufklärung”, in NEUGEBAUER-WÖLK, Aufklärung und Esoterik, S. 413.

83 Ibid., S. 414.

84 Cassirer, Philosophie der Aufklärung, S. 70. 
eines Gutachtens von Emanuel Antistes Merian von der Christentumsgesellschaft in Basel sowie in Württemberg wegen der apokalyptischen Theoreme und nicht zuletzt wegen der Spekulationen über den Hades verboten ${ }^{85}$. Schaut man sich jedoch Jung-Stillings Gegenargumente aus der Apologie an, so wird eine erstaunliche Diskontinuität deutlich. Denn er kritisiert explizit das Abweichen der Reformatoren von der für ihn zentralen Idee des Fegefeuers ${ }^{86}$, ohne selber dessen katholische Ausformulierung akzeptieren zu wollen. Anders gesagt versucht er einen Balanceakt zwischen Protestantismus und Katholizismus: die katholische Auffassung des Fegefeuers ist für ihn ein Irrtum der "Päpstischen" Christentum angemessen:

\begin{abstract}
Dass meine Lehre vom Hades, oder einer Reinigung nach dem Tod, von den ersten Zeiten an, bis auf die Reformation allgemeine Lehre der Christenheit oder der allgemeinen Kirchen gewesen, wird kein Sachkundiger leugnen. Da aber späterhin in der römischen Kirche ein Fegefeuer daraus gemacht wurde, aus dem man sich durch Seelenmessen, welche teuer bezahlt wurden, erlösen konnte, so hatten die Reformatoren ganz recht, dass sie das Fegefeuer aus ihren Glaubensartikeln verbannten, aber die Lehre der ersten Kirche von den fortdauernden Wirkungen des Erlösungswerks Christi nach dem Tod hätten sie beibehalten sollen ${ }^{88}$.
\end{abstract}

Damit steht nun aber der bis in seine Gegenwart reichende, die Menschen bis ins Jenseits verfolgende Anti-Humanismus der Reformatoren auf dem Spiel, dem sich Jung-Stilling eindeutig widersetzt:

[...] ich würde auch in meinen Schriften diesen Punkt nicht berühren, wenn uns die Philosophen und Neologen nicht den gegründeten Vorwurf machten, unsre Religion enthalte Lehren, welche die Würde des höchsten Wesens entehren und Gott zu einem Tyrannen machten, der seine Freude an den Qualen seiner Geschöpfe habe. Welcher Fürst wird ein Kind, um einiger jugendlicher Fehler willen auf lebenslang in einen schrecklichen Kerker verdammen? Und Gott, die ewige Liebe, sollte einen Menschen, sein Geschöpf, wenn er auch über hundert Jahre sündigte, mit einer Strafe belegen, die Myriaden Jahre dauerte, und wenn diese vorüber wären, wieder von vorne anfing! ${ }^{89}$

Hier reagiert Jung-Stilling auf seine Art auf die Kritik der Neologen an der Orthodoxie: er akzeptiert weder ihre Methode noch ihre Schlussfolgerungen, sondern er setzt ihnen seine Spekulation über den Hades entgegen. In dem Sinne bleibt er also konsequent und vermeidet jede Anwendung rationaler Methoden im Bereich des Glaubens. Gleichzeitig nimmt er jedoch durch seine Konzeption des Hades die Theorie der Apokatastasis Panton auf, so wie

85 Martin BRECHT / Klaus DePPERMANN (Hg.), Der Pietismus im achtzehnten Jahrhundert, Göttingen, Vandenhoeck \& Ruprecht, 1995, S. 737-738.

86 Jung-Stilling, Apologie der Geisterkunde, S. 30.

87 Ibid., S. 55.

88 Ibid., S. 42.

89 Ibid., S. 56 
sie in den vierziger Jahren des 18. Jahrhunderts von Vertretern der radikalen Frühaufklärung wie Johann Wilhelm Petersen und Ludwig Gerhard im Fahrwasser von Balthazar Bekker ${ }^{90}$ vertreten wurde ${ }^{91}$. Dass er damit trotz seines Status' als charismatischer Vordenker der Erweckungsbewegung ${ }^{92}$ den Protest der Baseler Christentumsgesellschaft auslöste, belegt wie langwierig, diskontinuierlich und fragmentarisch die Transformation von Wissen vor sich geht. Gleiches gilt für Jung-Stillings Rückgriff auf Positionen der radikalen Aufklärung: seine Verteidigung von Erbsünde und Apokatastasis Panton gleicht einer Quadratur des Kreises und beweist, dass gerade im Bereich der Wissenszirkulation eindeutige und dauerhafte Zuweisungen unmöglich sind.

90 Balthasar BEKKER, De Betoverde Wereld, Amsterdam 1691 ; deutsche Übersetzung Die bezauberte Welt, Amsterdam, 1693.

91 Vgl. KiTTSTEINER, "Abschaffung des Teufels", S. 65. Zur radikalen Frühaufklärung in der Nachfolge Spinozas vgl. Jonathan ISRAEL, Les Lumières radicales, Paris, éditions Amsterdam, 2005.

92 Vgl. die sympathisierende Darstellung von Jung-Stillings Publizistik bei ScHWINGE, Jung-Stilling als Erbauungsschriftsteller. $\mathrm{Zu}$ den Beziehungen zwischen der Christentumsgesellschaft und der Erweckungsbewegung vgl. Ulrich GÄBLER (Hg.), Der Pietismus im 19. und 20. Jahrhundert, Göttingen, Vandenhoeck \& Ruprecht, 1994, S. 87-244. 